Article

\title{
All-Fiber Hyperparametric Generation Based on a Monolithic Fiber Fabry-Pérot Microresonator
}

\author{
Kunpeng Jia $₫$, Xiaohan Wang, Jian Guo, Xin Ni, Gang Zhao *, Zhenda Xie * and Shining Zhu \\ National Laboratory of Solid State Microstructures, School of Electronic Science and Engineering, \\ School of Physics, College of Engineering and Applied Sciences, and Collaborative Innovation Center of \\ Advanced Microstructures, Nanjing University, Nanjing 210093, China; njujkp@gmail.com (K.J.); \\ xhwang174@gmail.com (X.W.); gj085208@163.com (J.G.); mf1823034@smail.nju.edu.cn (X.N.); \\ zhusn@nju.edu.cn (S.Z.) \\ * Correspondence: zhaogang@nju.edu.cn (G.Z.); xiezhenda@nju.edu.cn (Z.X.)
}

Received: 24 July 2020; Accepted: 28 September 2020; Published: 10 October 2020

\begin{abstract}
Hyperparametric oscillation is essential for coherent optical signal generation in a broad wavelength range. Integration in a compact system, such a broadband light source, is of special interest for practical applications requiring field-deployable spectroscopy devices. Here we demonstrate an all-fiber hyperparametric oscillation source based on four-wave mixing in a high-Q fiber Fabry-Pérot (FFP) microresonator. Assisted by the Raman effect, the generated optical signal spans over $400 \mathrm{~nm}$ with fine line-to-line spacing of $667 \mathrm{MHz}$. The compatibility of this FFP microresonator enables a robust and reliable all-fiber system through a splicing technique and fiber connectors. Such a plug-and-play platform is convenient and efficient for broad applications in optical communications and spectroscopy.
\end{abstract}

Keywords: four-wave mixing; hyperparametric optical oscillation; fiber Fabry-Pérot microresonator; Raman effect

\section{Introduction}

Hyperparametric oscillation based on four-wave mixing (FWM) in materials with third-order nonlinearity has attracted the attention of many researchers, as this nonlinear effect can convert a single-frequency pump laser to new discrete lasers [1-5], which is essential for applications like optical fiber communications, laser spectroscopy, and optical processing. In recent years, hyperparametric oscillation and FWM have been proven for use in optical frequency comb (OFC) [6-10] generation in Kerr microresonators, providing new access to equidistant frequency grids and optical cycles on the femtosecond time scale. These broadband OFCs are traditionally generated from mode-locked femtosecond lasers, which have been successfully deployed in a variety of fields like optical clocks, gas monitoring, precision spectroscopy, and photonic analog-to-digital converters [11-14], with unprecedentedly accurate standards in both the frequency and time domains. However, due to the bulky and costly table-top setup of mode-locked femtosecond lasers, their applications are mostly confined to laboratories. The OFC source based on FWM in Kerr microresonators is a promising candidate for a compact optical frequency standard $[8,9,14]$. The strong mode confinement and high finesse of microresonators enhance the nonlinear interactions in the FWM process, which have been demonstrated in a large number of platforms such as microrings [15], microdisks [16], and microspheres [17]. However, the quality factor $(\mathrm{Q})$ of these chip-scale microresonators is mainly limited by the propagation loss resulting from surface roughness and material absorption [18]. That makes it difficult to realize high-Q microresonators with a length of $100 \mathrm{~s} \mathrm{~mm}$, corresponding to repetition rates down to hundreds of megahertz, while $0.1-10 \mathrm{GHz}$ comb spacing is important to relax 
the requirements on comb processing electronics and is of special interest for applications such like dual-comb spectroscopy. Moreover, the pump light is coupled into these microresonators through the evanescent field typically from bus waveguides, tapered fibers, or prisms. Challenges lie in the fact that such a scheme requires either careful mode field overlap management or sophisticated free-space alignment, which may be unfavorable from a practical applications point of view.

In a monolithic fiber microresonator, direct pump light injection through end face coupling can be easily realized with low-cost commercial fiber connectors [19]. Such plug-and-play operation is expected to improve the stability and reduce the size and cost of the entire system. In addition, the quality factor of the fiber microresonator is mainly defined by the diffraction loss at the end faces, considering the negligible propagation loss of optical fiber. Thus, the fiber microresonator is a promising platform for Kerr OFC generation with a small footprint entering a frequency regime more typical of mode-locked lasers.

In this work, we demonstrate FWM-based hyperparametric generation in a wide spectral span from a fiber Fabry-Pérot (FFP) microresonator. Highly nonlinear fiber (HNLF) is fabricated with fine polishing and a dielectric coating for efficient hyperparametric oscillation. Continuous-wave (cw) pump light is coupled into the FFP microresonator through directly connected ceramic ferrules in a ceramic sleeve, as shown in Figure 1a, with an insertion loss below $0.5 \mathrm{~dB}$. The whole setup is fiber-based and all components, including the tunable laser, polarization controller, erbium-doped fiber amplifier (EDFA), and isolator, are connected by fiber splicing or using fiber connectors. Compared with the conventional evanescent field coupling method, such a configuration greatly simplifies the coupling steps and can well resist external mechanical disturbances coming from the environment. All in all, driven by a cw pump laser, FWM can be generated from our FFP microresonator with a fine spectrum tooth of $667 \mathrm{MHz}$. With the assistance of stimulated Raman scattering in the FFP microresonator $[16,20,21]$, the spectral span is further extended to over $400 \mathrm{~nm}$. It is worth noting that such broadband hyperparametric generation with sub-gigahertz line-to-line frequency separation is rarely achieved in a microresonator due to its propagation-limited $\mathrm{Q}$, as mentioned above.

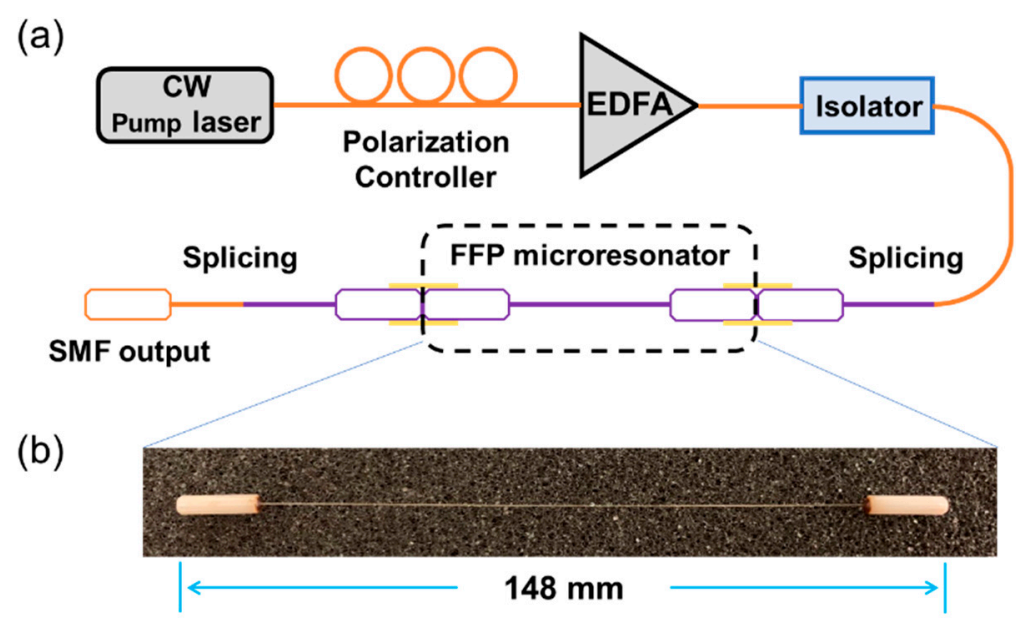

Figure 1. (a) Experimental setup. CW: continuous-wave; EDFA: erbium-doped fiber amplifier; SMF: single-mode fiber. The pump source, polarization controller, isolator, and Kerr microresonator are all fiber-based, resulting in low-loss fiber-to-fiber connection. (b) A picture of the 148-mm-long fiber Fabry-Pérot (FFP) microresonator. Fiber ends are mounted in ceramic ferrules, finely polished, and coated with dielectric mirror to form a high-Q microresonator.

\section{Experiment Setup and FFP Microresonator}

The experimental setup is shown in Figure 1a. The light from a C-band cw tunable pump laser (CTL1550, Toptica, Graefelfing, Germany) goes through an in-line fiber polarization controller and then is amplified in an EDFA. A fiber isolator is applied subsequently to prevent feedback light. Then, 
a short section of HNLF with a ceramic ferrule is fused with the output port of the isolator as an input coupler for the FFP microresonator. The splicing loss can be controlled to be less than $2 \mathrm{~dB}$. The length of HNLF was carefully tailored to be few centimeters to avoid nonlinear effects. Due to the identical HNLF used for the input coupler and FFP microresonator, the insertion loss is as low as $0.5 \mathrm{~dB}$ (or $10 \%$ ), ensuring high pump energy utilization. Finally, the output light of the FFP microresonator is coupled out by spliced HNLF-SMF (single-mode fiber) for the following measurement and analysis.

Our FFP microresonator was fabricated with a 148-mm-long HNLF (Zero-Slope, OFS, Norcross, GA, USA) (Figure 1b) with near-zero dispersion at $1550 \mathrm{~nm}$ and an effective mode area of $12.4 \mu^{2}$. Both ends of the HNLF were mounted in ceramic ferrules and finely polished. The Fabry-Pérot cavity was formed by the dielectric coating on both fiber ends with reflectivity of $99.5 \%$ at $1550 \mathrm{~nm}$. The quality factor of the FFP microresonator was characterized by scanning the frequency of the tunable laser over the resonances of the FFP microresonator. As shown in Figure 2a, the free spectral range (FSR) of our FFP microresonator was measured to be $667 \mathrm{MHz}$, which matches well with the $148 \mathrm{~mm}$ cavity length. The linewidth of the resonance was fitted to be $2.4 \mathrm{MHz}$, as shown in Figure $2 \mathrm{~b}$. For a high-Q microresonator, the quality factor can be calculated as $Q=\frac{v_{0}}{\Delta v}$, where $v_{0}$ is the center frequency of the resonant peak and $\Delta v$ is the linewidth of the resonance. Thus, our FFP microresonator has a $Q$ value of $8.1 \times 10^{7}$ at $1550 \mathrm{~nm}$. Similar designs of such FFP microresonators have been applied for Brillouin-enhanced Kerr comb generation [5] and pulse-driven temporal soliton generation [19]. However, neither of these designs could achieve a cw-pumped broadband spectrum as we demonstrate here, which is essential from a practical applications point of view.

(a)

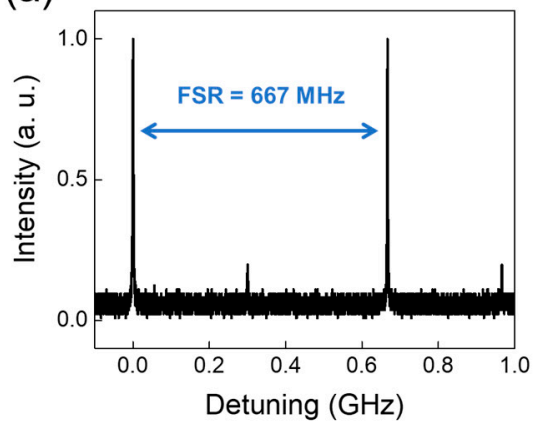

(b)

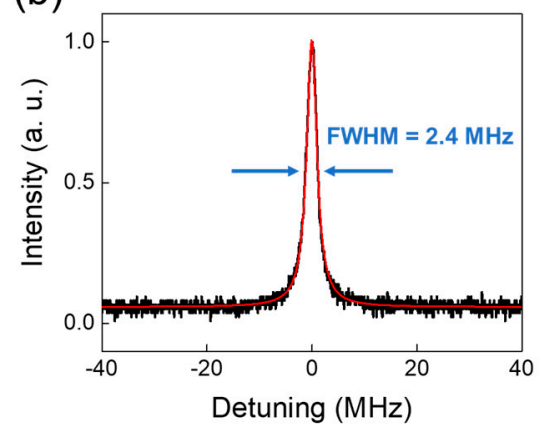

Figure 2. (a) The cold-cavity transmission signal when scanning a tunable laser across a resonance of around $1550 \mathrm{~nm}$, showing a $667 \mathrm{MHz}$ free spectral range (FSR). Two polarization modes are present, which originate from stress-induced birefringence resulting from the fiber ceramic ferrules, and the frequency detuning of the two modes is $301 \mathrm{MHz}$. In experiments, one of them can be fully suppressed using a polarization controller to ensure that only one polarization mode is excited in the FFP microresonator. (b) The transmission signal of one resonance. The linewidth was measured to be $2.4 \mathrm{MHz}$, which corresponds to a $\mathrm{Q}$ factor of $8.1 \times 10^{7}$.

\section{Hyperparametric Generation}

The pump laser was set at $1550 \mathrm{~nm}$ and tuned into deep resonance of the FFP microresonator from the blue-detuned side. The output spectra were recorded using an optical spectrum analyzer (OSA, AQ6375 YOKOGAWA). The threshold of four-wave mixing was measured to be $280 \mathrm{~mW}$. When the pump power was increased to $1.74 \mathrm{~W}$, Stokes and anti-Stokes Raman peaks were generated at the first and second order (Figure 3a). Note that the Stokes and anti-Stokes spectral lines are asymmetric in power due to the imperfect dielectric mirror coatings, which feature higher reflectivity at longer wavelength, thus the quality factor. The wavelength separation of the pump laser and the first-order Stokes Raman peak was measured to be $109.6 \mathrm{~nm}$. It is worth noting that primary comb lines can also be observed when the pump is just tuned into the cavity resonance. However, due to the low intra-cavity power and the weak thermal lock effect in the fiber resonator, the primary comb state is not stable enough for a complete spectral measurement. Sidebands around the pump and Raman peaks 
were generated via a four-wave mixing process (Figure $3 \mathrm{~b}$ ) with pump power of $3.5 \mathrm{~W}$, and they started to merge when the pump power was increased to $5.41 \mathrm{~W}$ (Figure 3c). When the pump power was further increased to $8.13 \mathrm{~W}$, the spectrum span extended to over $400 \mathrm{~nm}$ with the assistance of cascaded stimulated Raman scattering (SRS) (Figure 3d). It is worth noting that cavity dispersion typically limits the bandwidth of the Kerr comb in FFP microresonators to be less than $100 \mathrm{~nm}[5,19]$. Here, due to the small FSR (667 MHz) of our FFP microresonator, the Raman gain spectrum, which is typically about $100 \mathrm{~nm}$ in bandwidth [22], can easily cover over 10,000 cavity resonance modes, resulting in efficient pump energy conversion to Raman peaks. The combination of both SRS and Kerr nonlinearity breaks the limitation of cavity dispersion on the spectral bandwidth. The RF beatnote signal was measured using an electrical spectrum analyzer (ESA, FSVA30 R\&S), and the results are shown in Figure 3e,f. A clear peak at $667 \mathrm{MHz}$ was captured with a resolution bandwidth (RBW) of $1 \mathrm{MHz}$.

(a)

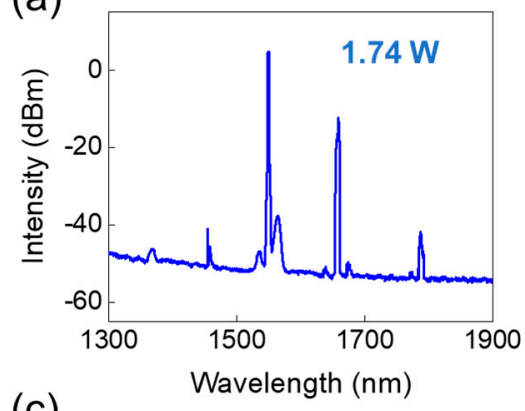

(c)



(e)



(b)

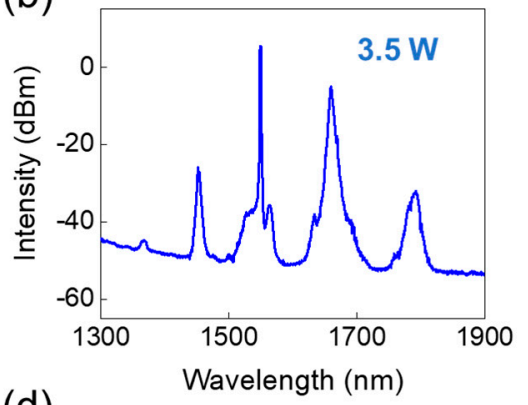

(d)



(f)

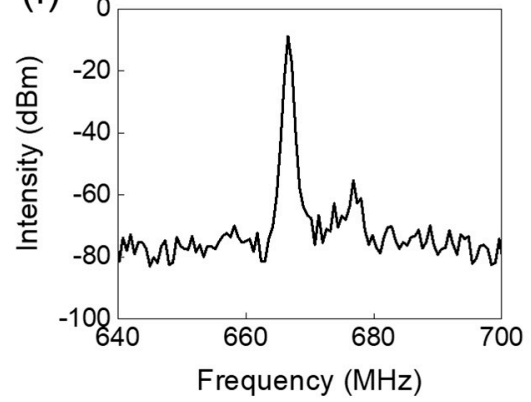

Figure 3. (a-d) Optical spectra of the FFP microresonator output with pump power set at $1.74 \mathrm{~W}, 3.5 \mathrm{~W}$, $5.41 \mathrm{~W}$, and $8.13 \mathrm{~W}$, respectively. The resolution of the optical spectrum analyzer (OSA) is insufficient to resolve $667 \mathrm{MHz}$ line-to-line spacing. The small slope of the spectrum baseline results from the uneven wavelength sensitivity of the detector and the Littrow light from blaze grating in the OSA. (e) RF beatnote spectrum, showing a repetition frequency of $667 \mathrm{MHz}$. RBW: resolution bandwidth. (f) Zoom-in view of the fundamental beatnote signal, showing a high signal-to-noise ratio (SNR) of about $50 \mathrm{~dB}$.

In order to show the spectral broadening due to the Raman effect more clearly, we performed numerical simulation using the Lugiato-Lefever equation (LLE) [23]. Here, the Raman effect was neglected; thus, pure Kerr nonlinearity was involved in the simulation. According to the manufacturer's published data, the dispersion of HNLF is $1 \mathrm{ps} /(\mathrm{nm} \cdot \mathrm{km})$ and the effective mode area is $12.4 \mu \mathrm{m}^{2}$. 
The pump wavelength was set to the deep effective red-detuned regime. The simulated spectrum is shown in Figure 4. The 30-dB bandwidth is less than $100 \mathrm{~nm}$, which is much narrower than the experimental Raman-assisted hyperparametric generation. Raman-induced spectral broadening for Kerr combs in microresonators has been demonstrated in several recent works [16,21,24]; this results from the complex interplay between Kerr, Raman, and all-order dispersion.

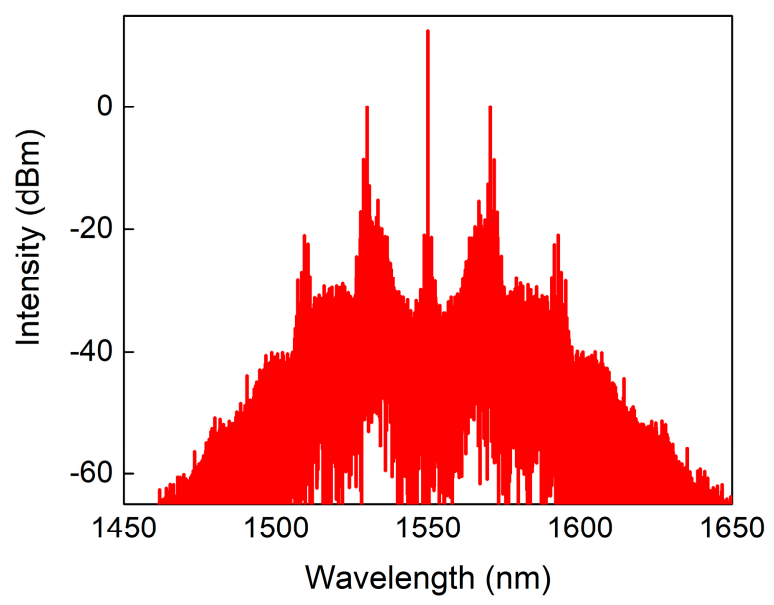

Figure 4. Simulated spectrum.

\section{Conclusions}

In conclusion, we demonstrated all-fiber hyperparametric generation based on a fiber Fabry-Pérot microresonator, which is compatible with today's widely used optical fiber systems. The high-quality FFP microresonator ensures strong self-phase modulation in a wide spectral range and eventually excites cascaded stimulated Raman scattering, which further extends the spectral bandwidth to over $400 \mathrm{~nm}$. The low transmission loss of HNLF enables a long cavity length of $148 \mathrm{~mm}$, resulting in fine line-to-line spacing of $667 \mathrm{MHz}$. This result may find broad application prospects in the fields of optical communication and spectroscopy. In the current situation, we demonstrated hyperparametric generation for the presentation of the FFP microresonator and all-fiber setup. In future work, we may further obtain a mode-locked frequency comb state and even a Stokes soliton comb with refined design of the cavity dispersion.

Author Contributions: Conceptualization, K.J. and X.W.; methodology, K.J.; software, K.J.; validation, K.J.; formal analysis, K.J.; investigation, K.J.; resources, K.J. and Z.X.; data curation, K.J., X.W., J.G. and X.N.; writing — original draft preparation, K.J.; writing—review and editing, K.J. and X.W.; visualization, K.J.; supervision, G.Z., Z.X. and S.Z.; project administration, G.Z., Z.X. and S.Z.; funding acquisition, G.Z., Z.X. and S.Z. All authors have read and agreed to the published version of the manuscript.

Funding: This research was funded by the National Key R\&D Program of China (2019YFA0705000, 2017YFA0303700), Key R\&D Program of Guangdong Province (2018B030329001), Leading-edge technology Program of Jiangsu Natural Science Foundation (BK20192001), and National Natural Science Foundation of China (51890861, 11690031, 11621091, 11627810, 11674169, 91950206).

Conflicts of Interest: The authors declare no conflict of interest.

\section{References}

1. Razzari, L.; Duchesne, D.; Ferrera, M.; Morandotti, R.; Chu, S.; Little, B.E.; Moss, D.J. CMOS-compatible integrated optical hyper-parametric oscillator. Nat. Photonics 2010, 4, 41-45. [CrossRef]

2. Ferrera, M.; Razzari, L.; Duchesne, D.; Morandotti, R.; Yang, Z.; Liscidini, M.; Yang, Z.; Liscidini, M.; Sipe, J.E.; $\mathrm{Chu}, \mathrm{S}$.; et al. Low-power continuous-wave nonlinear optics in doped silica glass integrated waveguide structures. Nat. Photonics 2008, 2, 737-740. [CrossRef]

3. Grudinin, I.S.; Yu, N.; Maleki, L. Generation of optical frequency combs with a $\mathrm{CaF}_{2}$ resonator. Opt. Lett. 2009, 34, 878-880. [CrossRef] 
4. Matsko, A.B.; Savchenkov, A.A.; Strekalov, D.; Ilchenko, V.S.; Maleki, L. Optical hyperparametric oscillations in a whispering-gallery-mode resonator: Threshold and phase diffusion. Phys. Rev. A 2005, 71, 033804. [CrossRef]

5. Braje, D.; Hollberg, L.; Diddams, S. Brillouin-Enhanced Hyperparametric Generation of an Optical Frequency Comb in a Monolithic Highly Nonlinear Fiber Cavity Pumped by a cw Laser. Phys. Rev. Lett. 2009, 102, 193902. [CrossRef] [PubMed]

6. Udem, T.; Holzwarth, R.; Hänsch, T.W. Optical frequency metrology. Nature 2002, 416, 233-237. [CrossRef] [PubMed]

7. Cundiff, S.T.; Ye, J. Colloquium: Femtosecond optical frequency combs. Rev. Mod. Phys. 2003, 75, 325. [CrossRef]

8. Kippenberg, T.J.; Holzwarth, R.; Diddams, S.A. Microresonator-based optical frequency combs. Science 2011, 332, 555-559. [CrossRef]

9. Del'Haye, P.; Schliesser, A.; Arcizet, O.; Wilken, T.; Holzwarth, R.; Kippenberg, T.J. Optical frequency comb generation from a monolithic microresonator. Nature 2007, 450, 1214-1217. [CrossRef]

10. Jones, D.J.; Diddams, S.A.; Ranka, J.K.; Stentz, A.; Windeler, R.S.; Hall, J.L.; Cundiff, S.T. Carrier-envelope phase control of femtosecond mode-locked lasers and direct optical frequency synthesis. Science 2000, 288, 635-639. [CrossRef]

11. Ghelfi, P.; Laghezza, F.; Scotti, F.; Serafino, G.; Capria, A.; Pinna, S.; Onori, D.; Porzi, C.; Scaffardi, M.; Malacarne, A.; et al. A fully photonics-based coherent radar system. Nature 2014, 507, 341-345. [CrossRef] [PubMed]

12. Khilo, A.; Spector, S.J.; Grein, M.E.; Nejadmalayeri, A.H.; Holzwarth, C.W.; Sander, M.Y.; Dahlem, M.S.; Peng, M.Y.; Geis, M.W.; DiLello, N.A.; et al. Photonic ADC: Overcoming the bottleneck of electronic jitter. Opt. Express 2012, 20, 4454-4469. [CrossRef] [PubMed]

13. Mahjoubfar, A.; Churkin, D.V.; Barland, S.; Broderick, N.; Turitsyn, S.K.; Jalali, B. Time stretch and its applications. Nat. Photon. 2017, 11,341-351. [CrossRef]

14. Papp, S.B.; Beha, K.; Del'Haye, P.; Quinlan, F.; Lee, H.; Vahala, K.J.; Diddams, S.A. Microresonator frequency comb optical clock. Optica 2014, 1, 10-14. [CrossRef]

15. Jung, H.; Xiong, C.; Fong, K.Y.; Zhang, X.; Tang, H.X. Optical frequency comb generation from aluminum nitride microring resonator. Opt. Lett. 2013, 38, 2810-2813. [CrossRef]

16. Fang, Z.; Luo, H.; Lin, J.; Wang, M.; Zhang, J.; Wu, R.; Zhou, J.; Chu, W.; Lu, T.; Cheng, Y. Efficient electro-optical tuning of an optical frequency microcomb on a monolithically integrated high-Q lithium niobate microdisk. Opt. Lett. 2019, 44, 5953-5956. [CrossRef]

17. Riesen, N.; Afshar, S.; François, A.; Monro, T.M. Material candidates for optical frequency comb generation in microspheres. Opt. Express 2015, 23, 14784-14795. [CrossRef]

18. Suh, M.G.; Vahala, K. Gigahertz-repetition-rate soliton microcombs. Optica 2018, 5, 65-66. [CrossRef]

19. Obrzud, E.; Lecomte, S.; Herr, T. Temporal solitons in microresonators driven by optical pulses. Nat. Photon. 2017, 11, 600-607. [CrossRef]

20. Yang, Q.F.; Yi, X.; Yang, K.Y.; Vahala, K. Stokes solitons in optical microcavities. Nat. Phys. 2017, $13,53-57$. [CrossRef]

21. Jung, H.; Gong, Z.; Liu, X.; Guo, X.; Zou, C.L.; Tang, H.X. Stokes and anti-Stokes Raman scatterings from frequency comb lines in poly-crystalline aluminum nitride microring resonators. Opt. Express 2019, 27, 22246-22253. [CrossRef]

22. Hollenbeck, D.; Cantrell, C.D. Multiple-vibrational-mode model for fiber-optic Raman gain spectrum and response function. JOSA B 2002, 19, 2886-2892. [CrossRef]

23. Coen, S.; Randle, H.G.; Sylvestre, T.; Erkintalo, M. Modeling of octave-spanning Kerr frequency combs using a generalized mean-field Lugiato-Lefever model. Opt. Lett. 2013, 38, 37-39. [CrossRef]

24. Zhu, S.; Shi, L.; Ren, L.; Zhao, Y.; Jiang, B.; Xiao, B.; Zhang, X. Controllable Kerr and Raman-Kerr frequency combs in functionalized microsphere resonators. Nanophotonics 2019, 8, 2321-2329. [CrossRef] 